\author{
Mariantonietta Savarese \\ Mario Guazzelli \\ Maria Pia Prudenzano \\ Mafalda Carnicelli \\ Marco Rossi \\ Valentina Cardinali \\ Sergio Genco \\ Paolo Lamberti \\ Paolo Livrea
}

\title{
Tertiary treatment for psychiatric comorbidity in headache patients
}

Published online: 20 July 2005

M. Savarese ( $₫) \cdot$ M.P. Prudenzano

M. Carnicelli • M. Rossi • V. Cardinali

S. Genco • P. Lamberti • P. Livrea

Headache Disorders Center,

Department of Neurological

and Psychiatric Sciences,

University of Bari,

Piazza G. Cesare 11, I-70124 Bari, Italy

e-mail: m.savarese@neurol.uniba.it

Tel.: +39-080-5592331/5478527

Fax: +39-080-5593079

M. Guazzelli

Psychology Clinic,

University of Pisa, Pisa, Italy

\begin{abstract}
The presence of significant and confounding psychiatric comorbidity is greater in patients attending headache clinics than in headache patients from the general population. The frequent comorbidity of headache with generalized anxiety disorder can take advantage of the administration of benzodiazepines. With regard to depression-related headache, it's wellknown that the antidepressive drugs can improve migraine as well as tension-type headache.

Antiepileptic drugs give one more good opportunity. The recognition of a psychiatric comorbidity is mandatory for an accurate manage-
\end{abstract}

ment of the patient beacause prevents the clinicians from using any drug that might be dangerous for a mysdiagnosed psychiatric disturbance and often permits to administer medications that can efficaciously control both headache and psychiatric disorders.
Key words Anxiety • Depression • Tension type headache $\cdot$ Migraine
The presence of significant and confounding psychiatric comorbidity is greater in patients attending headache clinics than in headache patients from the general population. Both tension-type headache (TTH) and migraine without aura are associated with psychiatric disorders in a great number of Italian patients (respectively, $77.8 \%$ and $56.4 \%$ ). The most recurrent cluster of psychiatric disorders was anxiety (52\% and $39.2 \%$ respectively), followed by mood disorders (36\% and 23\%) and, to a lesser degree, somatoform disorder (21\% and $21.6 \%)$ [1, 2]. Psychiatric comorbidity was more frequent in the chronic than in the episodic form of TTH [1]; on the other hand, it is well known that psychological factors may play an important role in both peripheral and central mechanisms of sensiti- sation that are in turn responsible for transformation of episodic headache into chronic headache.

The recognition of a psychiatric comorbidity is mandatory for accurate management of the patient because it prevents clinicians from using a drug that might be dangerous for a misdiagnosed psychiatric disturbance and often permits medication that can efficaciously control both headache and psychiatric disorders.

So, the association of depressive symptoms has to be taken into account when deciding to start a migraine prophylaxis with flunarizine or beta-blockers in worsening mood disorders.

With regard to depression-related headache, it is well known that antidepressive drugs can improve migraine as 
well as TTH by an increase of serotoninergic and/or noradrenergic pool that exerts a descending inhibiting modulation of pain at the level of spinal dorsal horn/trigeminal neurons. Antidepressive drugs can control also anxiety disorders possibly associated with depressive symptoms: this is true not only for the tricyclic antidepressants (TCAs) but also for the serotonin selective reuptake inhibitors (SSRIs), which lack the sedative effects of the TCAs but have a differential advantage in treating anxiety within depression probably by means of an induced normalisation in processing information; certainly some differences exist in sedative side effects of novel antidepressants: for example, within SSRIs, fluvoxamine and sertraline are more sedative and within mixed norepineprhine-serotonin reuptake inhibitors (NSRI), mirtazepine has strong sedative properties; so, only one of these medications could improve at the same time pain, depression and anxiety (Fig. 1). The anti- depressive properties of anti-epileptic drugs (AEDs) and the rapidly rising popularity of AEDs in preventive migraine treatment give one more good opportunity for a pharmacological approach to both diseases by only one drug with a greater safety when using new AEDs such as topiramate. Particularly, valproate can be useful when the depressive syndrome is of sufficient severity to satisfy the criteria for major depression.

In the cluster of anxiety-related headaches, generalised anxiety disorder (GAD) represents the most frequent disorder. Benzodiazepines (BZDs) act at specific BZD receptors to enhance the binding of the inhibitory neurotransmitter GABA with a following improvement of anxious symptoms; but GABAergic neurons have been also shown in the dorsal horn and in trigeminal caudalis nucleus to receive nociception inputs, so as to exert presynaptic control on ascending pain [3]; therefore, BZDs can raise the

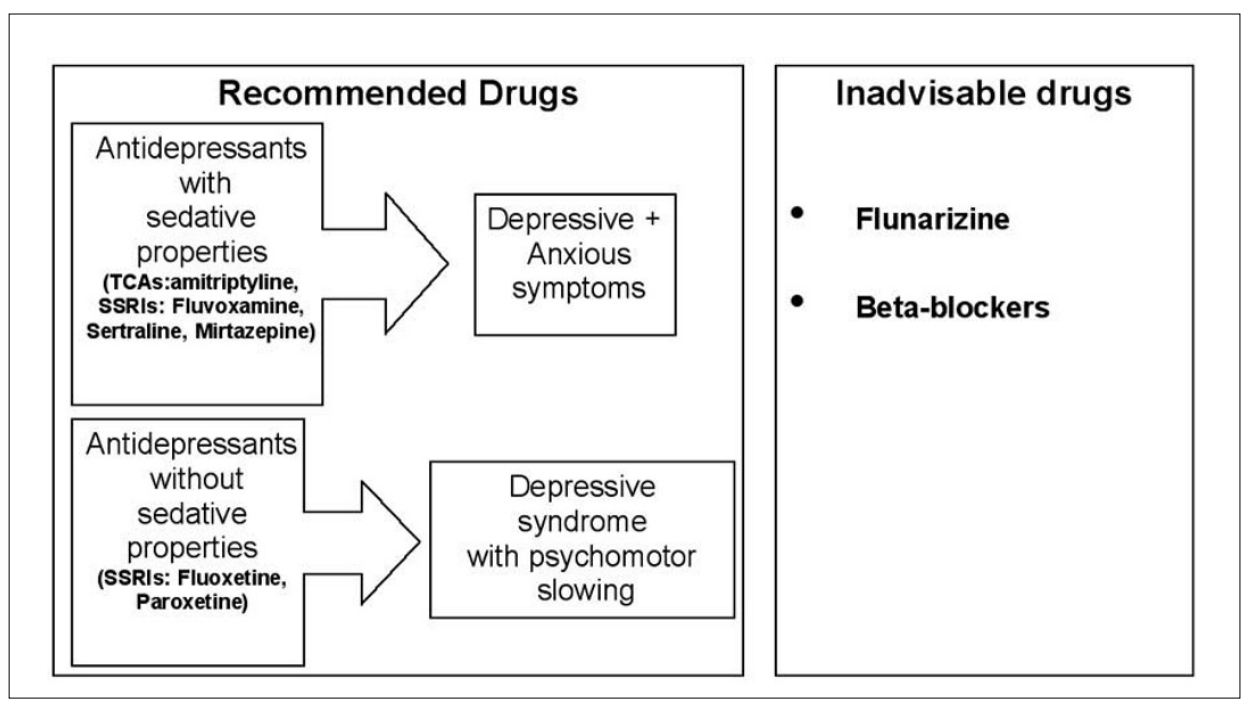

Fig. 1 Preventive treatment in headache associated with depressive syndrome

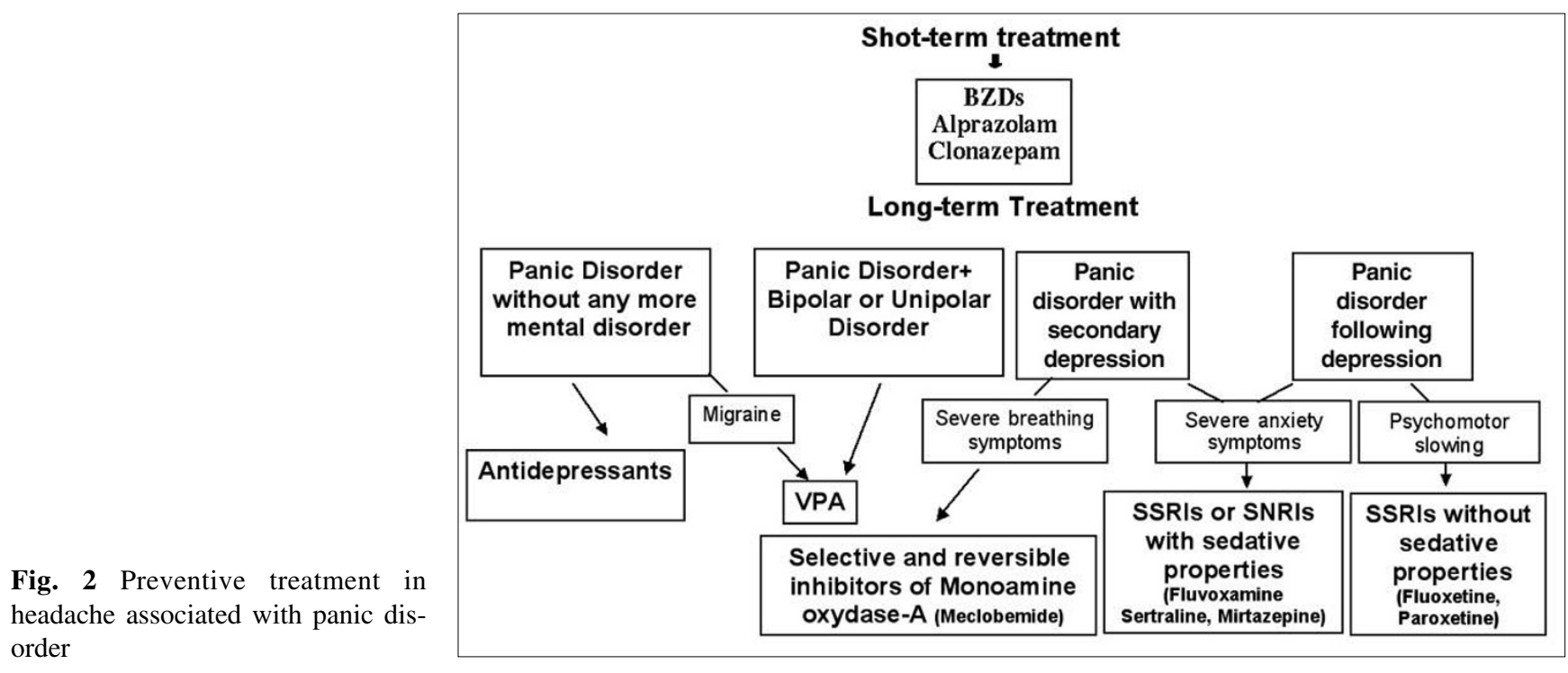


pain threshold due to a GABAergic modulation of endorphinergic or serotoninergic/noradrenergic activity; moreover, in TTH, myorelaxation potentiation associated with some BZDs like diazepam may play an important role in the improvement of pain. So, both GAD and TTH can take advantage of the administration of BZDs, but long-term therapy with BZD is not eligible in the aim of avoiding tolerance. As regards the preventive treatment of this association, the beta-blockers can aid both migraine and GAD because of intrinsic anxiolytic property of these medications; flunarizine can be used too, owing to its sedative side effect.

GAD is not the only anxiety disorder which may be related to headache. Panic disorder has been shown to be associated with TTH as well as migraine $[1,2]$. In the first case, the short-term intake of BZDs like alprazolam can be efficacious in both disorders; in the second case, valproate (VPA) can be used due to its property of inhibiting migraine "hyperexcitabilty state" by means of a potentiation of glutamate-decarboxylase enzyme and resulting decrease of glutamate pool. As a great number of patients with panic disorder suffer also from depression, the association of panic disorder with TTH or migraine can show benefits when treated with TCAs or SSRIs/NSRIs. The SSRIs are first choice medications also for long-term treatment of TTH+panic disorder without mood disorders, because positive results have been seen in the treatment of panic disorder with these drugs in agreement with the demonstrated efficacy on anxiety symptoms. The associa- tion between migraine and panic disorder and bipolar disorder or unipolar disorder can be treated with VPA, which is effective in the control of panic (Fig. 2).

Obsessive-compulsive disorder was not found in TTH patients and was found only in migraineur females $(5 \%)$; the administration of SSRI can improve both headache and this anxiety disorder; when obsessive-compulsive disorder is also associated with a major depressive disorder, the treatment is different according to whether this is a unipolar disorder or a bipolar disorder; in the first case, the dose of SSRI can be lower and a replacement of SSRI with NSRI may be considered; in the second case, the intake of SSRI must be stopped and an antimaniacal treatment must be started.

When psychiatric comorbidity of TTH and migraine consists of somatoform disorder, the antidepressive drugs are the medications of first choice.

In conclusion, it becomes possible to draw up some useful flow-charts of pharmacological management for psychiatric comorbidity in headache patients.

A good knowledge of the right management of psychiatric comorbidity in headache represents the only card which may be played by clinicians in the aim of breaking the "psycho-neuro-biochemical loop": really, as any psychological disorder can affect the onset and the course of somatic disorders which in turn can increase psychiatric vulnerability [4], the misdiagnosis of a psychiatric comorbidity might lead to a progressive worsening of headache or to its dangerous transformation into a chronic form.

\section{References}

1. Puca F, Genco S, Prudenzano MP, Savarese M, Bussone G, D'Amico D, Cerbo R, Gala C, Coppola MT, Gallai V, Firenze C, Sarchielli P, Guazzelli M, Guidetti V, Manzoni G, Granella F, Muratorio A, Bonuccelli U, Nuti A, Nappi G, Sandrini G, Verri AP, Sicuteri F, Marabini S (1999) Psychiatric comorbidity and psychosocial stress in patients with tension-type headache from headache centers in Italy. Cephalalgia 19(3):159-164
2. Puca F, Genco S, Prudenzano MP, Savarese M, Bussone G, D'Amico D, Cerbo R, Gala C, Coppola MT, Gallai V, Firenze C, Sarchielli P, Guazzelli M, Guidetti V, Manzoni G, Granella F, Muratorio A, Bonuccelli U, Nuti A, Nappi G, Sandrini G, Verri AP, Sicuteri F, Marabini S (2000) Psychological and social stressors and psychiatric comorbidity in patients with migraine without aura from headache centers in Italy: a comparison with tension-type headache patients. J Headache Pain 1:17-25
3. Malcangio M, Bowery NG (1996) GABA and its receptors in the spinal cord. Trends Pharmacol Sci 17:457-462

4. Pietrini P, Guazzelli M (1997) Life events in the course of chronic diseases: a psychological myth or a psycho-neuro-biochemical loop? Clin Exp Rheumatol 15:125-128 